\title{
Renal Anastomosing Hemangioma Simulating Angiosarcoma as a Second Primary in a Patient with Breast Cancer
}

\section{Daniel Fernando Méndez López, Jhonatan Gómez Domínguez, Jorge Antonio Rojas González, Marco Antonio Ortiz Jiménez, Omar Santos Moreno}

Department of Radiology in the Governmental Hospital "Hospital de Especialidades 5 de Mayo" (ISSSTEP), Puebla,

Mexico

Email: jhonatangomdom@gmail.com

How to cite this paper: Méndez López, D.F., Gómez Domínguez, J., Rojas González, J.A., Ortiz Jiménez, M.A. and Santos Moreno, O. (2021) Renal Anastomosing Hemangioma Simulating Angiosarcoma as a Second Primary in a Patient with Breast Cancer. Open Journal of Urology, 11, 466-473. https://doi.org/10.4236/oju.2021.1112047

Received: October 2, 2021

Accepted: December 17, 2021

Published: December 20, 2021

Copyright $\odot 2021$ by author(s) and Scientific Research Publishing Inc. This work is licensed under the Creative Commons Attribution International License (CC BY 4.0).

http://creativecommons.org/licenses/by/4.0/

\begin{abstract}
Anastomosing hemangioma is a type of tumor lesion of benign behavior scarcely documented, it has been observed in renal parenchyma, testicles, ovaries and adrenal glands, it is usually prone to perirenal and renal medullary adipose tissue. In this case report, we present an anastomosed hemangioma in the right kidney of a 33-year-old woman, with previous coexistence of breast cancer, in which an unusual behavior was detected, with characteristics of vascular lesion, in this case we report: The classic conditions, its heterogeneous nature and the vascular alterations that presents in different imaging modalities.
\end{abstract}

\section{Keywords}

Anastomosing Hemangioma, Kidney, Tumor Lesion, Breast Cancer

\section{Introduction}

Hemangiomas are common mesenchymal tumors, which manifest mainly on the skin, soft tissues, and viscera. The existence of reports about renal vascular tumors is scarce, the literature of angiosarcomas and hemangiomas are limited to some series of reports and clinical cases, even when renal vascularity is considerable, receiving $20 \%$ of cardiac output [1].

Anastomosing hemangioma is a tumor-like lesion of vascular etiology and benign behavior [2]. It was first described in 2009 by Montgomery and Epstein as a vascular lesion that involves the renal parenchyma and mimics angiosarcoma. The composition of this type of hemangioma is characterized by irregularly anastomosed sinusoidal vascular spaces, surrounded by endothelial cells with mi- 
nimal atypia. Generally described with a tack morphology, with minimal marginal invasion and a stroma, formed of spindle cells that express the immunohistochemical marker CD34.

Different clinical studies have been described in: testicles, gastrointestinal tract, ovaries and adrenal glands. Anastomosing hemangioma has a propensity for perirenal adipose tissue and renal medulla [3] [4]. Renal hemangiomas are commonly unilateral and isolated, however in $12 \%$ of clinical researches, they can show multiple hemangiomas [5]. It mainly affects the young population, with no specific predilection for gender. The usual characteristic symptoms are hematuria and renal colic, however incidental findings are common in asymptomatic patients [6].

\section{Image Assessment}

The diagnostic through ultrasonography has a limited literature, because anastomosing hemangioma is a variable type of tumor lesion, and ultrasound examination usually shows different echogenicity ranges [7]. In our case report, a tumor-type lesion is documented with a slight increase in echogenicity, compared to the renal parenchyma, associated with calcifications and peripheral vascularity (Figure 1).

By means of computed tomography, hemangiomas are described as neoplasms with a circular appearance, with a low heterogeneous density of soft tissues, in the arterial phase they usually manifest a septal enhancement that persists during the venous phase [8].

Considering the characteristics detected by renal magnetic resonance imaging, anastomosing hemangioma is described as a lesion with a rounded, well-defined morphology [9], hypointense in T1 sequence and hyperintense in T2 sequence, and with substantial enhancement to the contrast medium [8].

\section{Case Report}

33-year-old woman with a three-year history of breast cancer; the patient received

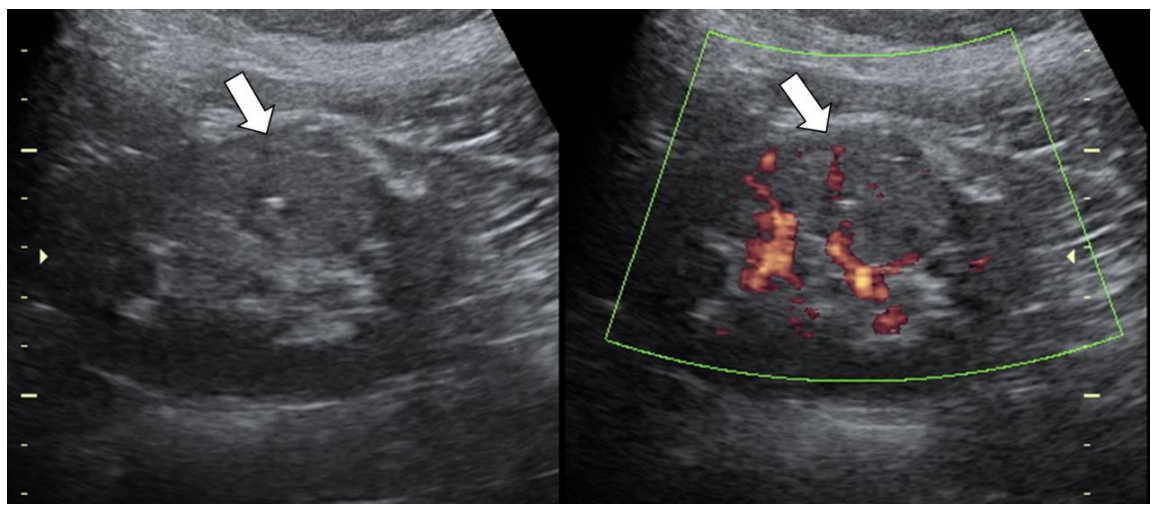

Figure 1. Gray scale ultrasound of the right kidney, longitudinal section showing a well-defined lesion (white arrows), slightly hyperechoic with internal calcification, peripheral vascularization was scrutinized via color Doppler. 
an initial treatment of four cycles of chemotherapy (epirubicin); subsequently, on December $2^{\text {nd }}$ of 2017, a radical mastectomy with axillary dissection was performed. Afterwards, a histopathological diagnosis of infiltrating ductal carcinoma was carried out, indicating a positive molecular subtype for estrogen and progesterone receptors.

In September of 2020, after 37 sessions of radiotherapy, she was monitored in order to provide follow-up; by means of thoracoabdominal computed tomography, with simple and contrasted phases; some characteristics of metastasis, local and generalized lymphadenopathy were found, as well as lesions suggestive of secondary deposits in the abdominal wall; as a discovery, a tumor lesion with soft tissue density was found in the lower pole of the right kidney, which has an appreciable enhancement to contrast in arterial phase, therefore, by means of the known diagnostic methods it was conjectured in mammary metastasis to the kidney, with a second primary lesion (angiosarcoma) (Figure 2).

Consequently, and due to the suspicion of a neoplastic process, the case was considered as a secondary deposit of a known primary by the oncology service.

In April of 2021, we optimized the acquisition method and computed tomography protocol, in this case report, we found that the renal lesion has a vascular compartment to the intravenous contrast, without changes in size and morphology, so it is considered indicative of benignity (Figure 2).

In complementation, an MRI was also performed, revealing an image with a

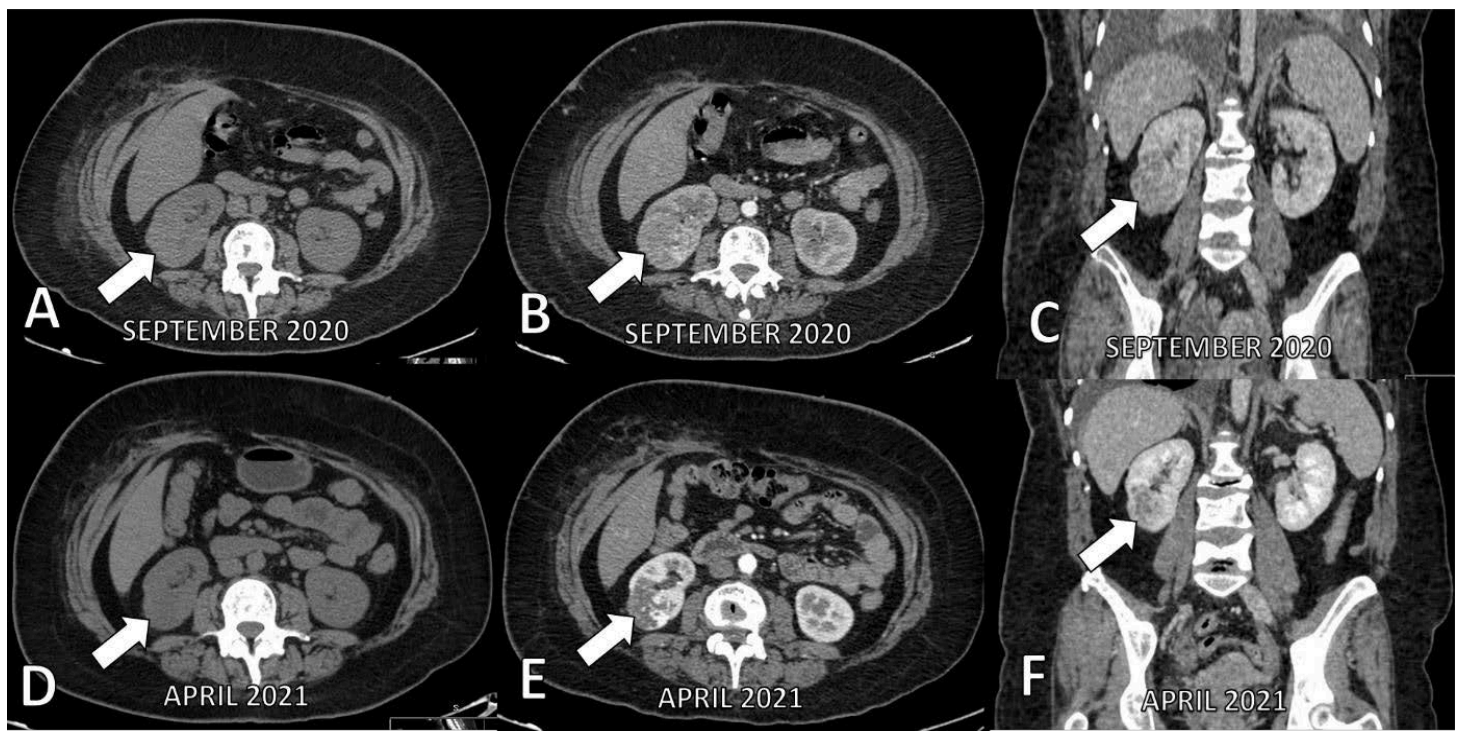

Figure 2. CT, performed in September of 2020, with the objective of screening the therapeutic response to breast cancer. (A) Simple phase axial view reveals the presence of a hypodense tumor-like lesion with soft tissue density, located in the lower pole of the right kidney. (B) After the administration of medium contrast in arterial phase, a heterogeneous enhancement with peripheral predominance is observed. (C) Coronal section reveals the persistence of heterogeneous enhancement throughout the venous phase. Because of the characteristics of the lesion shown in the radiological report, it is surmised as a second primary tumor, a renal cell carcinoma versus an angiosarcoma. A second monitoring was performed in April of 2021. (D) and (E) show the presence of a neoplastic lesion with considerable peripheral enhancement. (F) Coronal section with a central filling of contrast medium in venous phase. 
mixed pattern in intensity, showing hyperintense areas in T2 sequence and hypointense in $\mathrm{T} 1$ sequence, as well as dotted/stippled areas located in the lower pole of the right kidney (Figure 3).

As a backup, an ultrasonography was performed in order to closely monitor patient's kidneys, revealing a rounded image in the lower pole of the right kidney, slightly hyperechoic in relation to the adjacent renal parenchyma, and with the presence of peripheral and central vascularity when using color Doppler (Figure 1).

The method selected consisted a percutaneous biopsy guided by ultrasonography, employing a coaxial device and a semi-automatic tru-cut biopsy gun of $14 \mathrm{~g}$. Obtaining a total of 4 sections, the analysis of the pathology department specified the following findings: probable subcapsular tissue damage, tubular atrophy and mild interstitial fibrosis, mild to moderate atherosclerosis with $25 \%$ vascular lumen compromised in some arteries; unfortunately, an optimal result was not achieved, however the diagnosis by biopsy revealed no evidence of malignancy (Figure 4).

It was decided to employ a percutaneous biopsy, in comparison with the majority of the reported cases in which a partial nephrectomy was opted for; however, the complexity of this method was confirmed, due to the prioritization of renal integrity over puncture the vascular component.

Subsequently, once there was no evidence of malignancy, the patient's protocol continued, with regular visits to the oncology department and control scans every 6 months, in which no signs of growth or indication of malignancy have been detected.

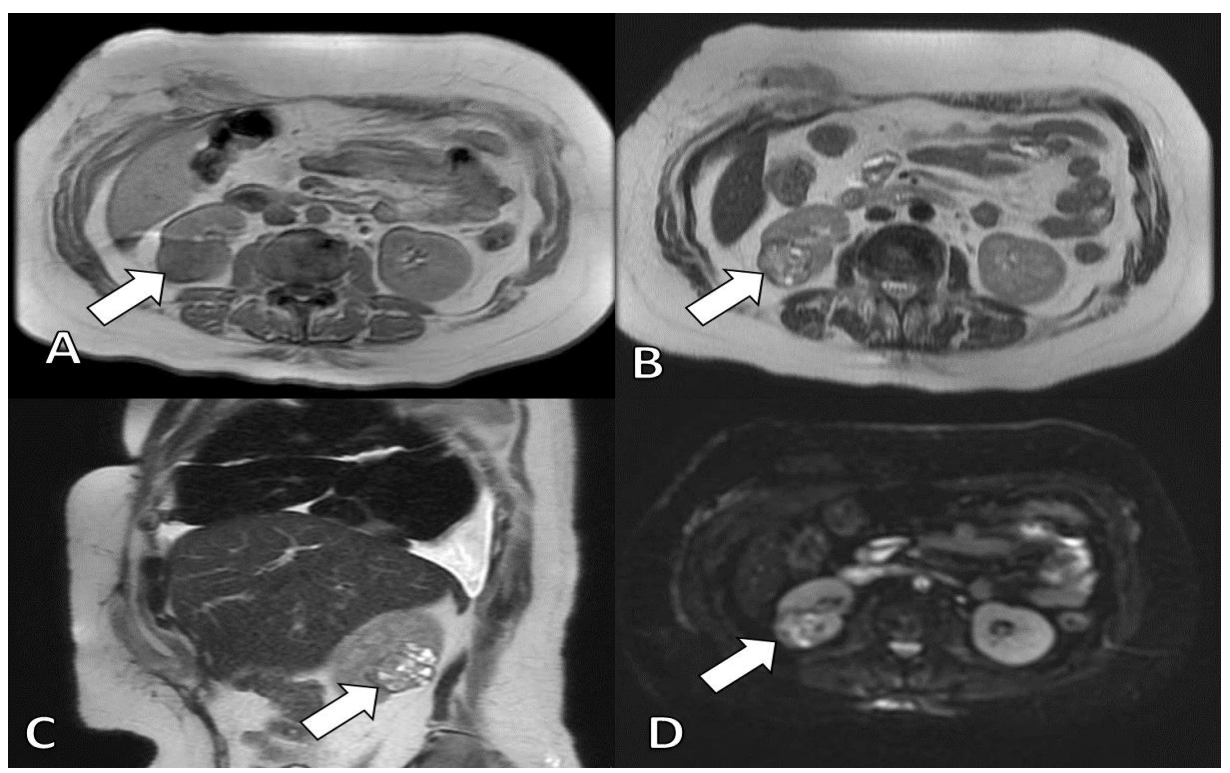

Figure 3. MRI, April of 2021, shows evidence of a mixed intensity pattern; (A) and (B) predominantly hypointense in T1 with hyperintense areas in T2; (C) also obtained dotted images that revealed absence of signal during vascular sequences, located in the lower pole of the right kidney; (D) T2 with fat saturation, with central hyperintensity. 


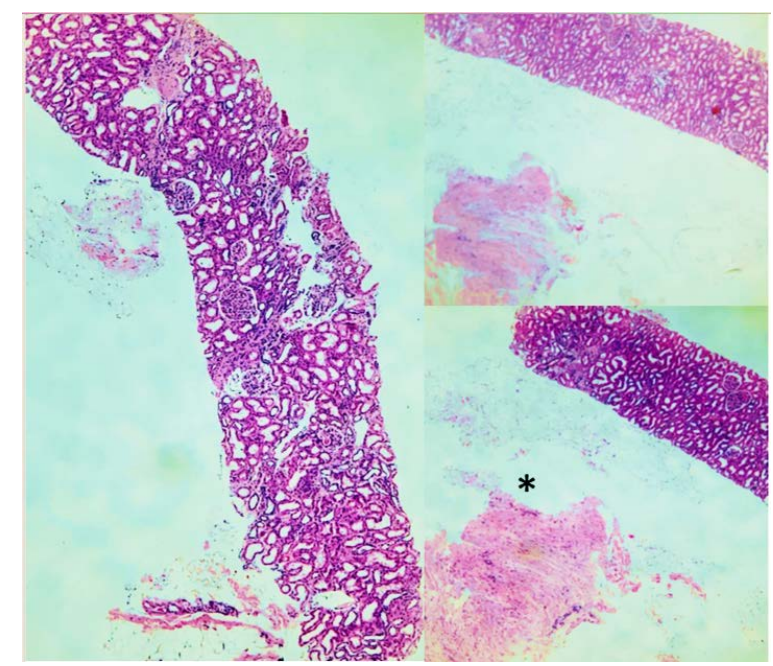

Figure 4. Hematoxylin and eosin (10x magnification) renal section with permeable proximal tubules and without glomerular abnormalities-Renal capsule $\left.{ }^{*}\right)$.

\section{Discussion}

Renal tumors of vascular origin are unusual, the most frequent subgroup consisting of benign hemangiomas.

Renal hemangiomas are usually detected in incidental circumstances and asymptomatic patients. In the symptomatic patient, the regular symptoms are hematuria and abdominal pain [10].

In the global knowledge, it is estimated that 200 cases are reported, but with limited radiological findings [1], consequently, we have a limited information about the radiological characteristics of the condition; although there are cases reported with erroneous diagnoses of renal cell carcinomas or angiosarcomas, a renal hemangioma appearing to be a metastasis deposit or a second primary tumor has not been specified in a patient with breast cancer [2].

According to the literature, renal hemangiomas generally have the presence of small or large injuries, with an average size of 2.1 centimeters, and a range of 1 to 6 centimeters [7] [11].

In this report, through ultrasonography, a well-defined tumor-like lesion with presence of peripheral vascularity was observed, which was achieved with the help of color Doppler (Figure 1). By means of computed tomography, a predominantly hypodense heterogeneous lesion with internal calcifications and with a substantial heterogeneous enhancement with vascular features (Figure 2). With the help of magnetic resonance imaging, a mixed intensity pattern with hyperintense areas in $\mathrm{T} 2$ and hypointense in $\mathrm{T} 1$ areas were detected in the lower pole of the right kidney (Figure 3). The imaging findings correspond to those reported in the literature [2] [4].

The differential diagnoses between benign and malignant vascular tumors is realized by histopathology [4], in this case, the possibility of an angiosarcoma or metastasis of the known primary was ruled out, by virtue of a later tomography performed to evaluate the therapeutic response, an improvement in the contrast 
phases was obtained, which allowed us an optimal identification of the lesion, in which a vascular behavior without important changes in size and morphology, features suggestive of benignity were found. On the other hand, vascular tumors of malignant origin such as angiosarcoma usually have an aggressive behavior with metastasis to the liver, lungs and bone at the time of diagnosis.

Regardless of the fact that anastomosing hemangioma is uncommon, its identification and separation from other malignant entities is essential, because a meticulous diagnosis offers the opportunity to provide a conservative treatment using selective embolization, in comparison to the use of a radical nephrectomy, which is often chosen as treatment [2].

Histopathological analysis reveals in this kind of tumor lesions, the presence of intravascular growth of the hemangioma, multilayers of cellular endothelium, and an absence of cellular atypia. It was determined to obtain biopsy by percutaneous needle puncture, taking into consideration the vascular pattern, the risk was reduced with the help of color Doppler, however, the result was not the convenient one, and only the increase of the level of vascular hyperplasia from mild to moderate with a compromise of $20 \%$ of the vascular lumen of the arterioles close to the glomerular hilum was documented (Figure 4).

\section{Conclusions}

Due to the fact that hemangioma is a barely reported disease, all the documentation of the general and radiological features detected by ultrasound, computed tomography and magnetic resonance imaging are very helpful. In addition, the prior coexistence of stage IV metastatic breast cancer is a consideration when relating and clarifying the diagnosis with histopathologic analysis. Although most reports are obtained through surgical procedures via partial or total nephrectomy, in this case report, it was determined to use an ultrasound-guided needle biopsy, which proved to be a convenient alternative, considering that in addition to being an affordable medical treatment, it offers results in less time, since it reduces a medical care process that for various circumstances can be delayed, finally, we consider this alternative less invasive, also leaves little or no scarring, and does not require exposure to ionizing radiation.

To our knowledge, this is one of the first reports in which ultrasonography, computed tomography and magnetic resonance imaging are documented in the same patient. We hope that this information will be useful in the clinical and radiological field, helping in the identification of subsequent renal hemangiomas.

\section{Acknowledgements}

The authors wish to thank Marco Antonio Ortiz Chávez for editorial assistance and translation with this manuscript, Lluvia Mixlitzin Alvarado Robles Miriam and Miriam Ixel Escamilla López for contacting and medical care for the patient in this case report. 


\section{Conflicts of Interest}

The authors declare no conflicts of interest regarding the publication of this paper.

\section{References}

[1] Brown, J.G., Folpe, A.L., Rao, P., Lazar, A.J., Paner, G.P., Gupta, R., et al. (2010) Primary Vascular Tumors and Tumor-Like Lesions of the Kidney: A Clinicopathologic Analysis of 25 Cases. The American Journal of Surgical Pathology, 34, 942-949. https://doi.org/10.1097/PAS.0b013e3181e4f32a https://pubmed.ncbi.nlm.nih.gov/20534992/

[2] Cheon, P.M., Rebello, R., Naqvi, A., Popovic, S., Bonert, M. and Kapoor, A. (2018) Anastomosing Hemangioma of the Kidney: Radiologic and Pathologic Distinctions of a Kidney Cancer Mimic. Current Oncology, 25, 220-223.

https://doi.org/10.3747/co.25.3927 https://pubmed.ncbi.nlm.nih.gov/29962849/

[3] Lappa, E. and Drakos, E. (2020) Anastomosing Hemangioma: Short Review of a Benign Mimicker of Angiosarcoma. Archives of Pathology \& Laboratory Medicine, 144, 240-244. https://doi.org/10.5858/arpa.2018-0264-RS https://pubmed.ncbi.nlm.nih.gov/30958692/

[4] Rubio Fernández, A., Díaz Delgado, M. and Hernández Amate, A. (2015) Múltiples hemangiomas anastomosantes y lesiones precursoras de carcinoma de células renales asociado a enfermedad renal quística adquirida. Revista Española de Patología, 48, 103-108. https://doi.org/10.1016/j.patol.2014.09.005 https://www.sciencedirect.com/science/article/abs/pii/S1699885514000956?via\%3Di $\underline{\text { hub }}$

[5] Lee, H.S., Koh, B.H., Kim, J.W., Kim, Y.S., Rhim, H.C., Cho, O.K., et al. (2000) Radiologic Findings of Renal Hemangioma: Report of Three Cases. Korean Journal of Radiology, 1, 60-63. https://doi.org/10.3348/kjr.2000.1.1.60 https://www.ncbi.nlm.nih.gov/pmc/articles/PMC2718141/

[6] Prasad, S.R., Surabhi, V.R., Menias, C.O., Raut, A.A. and Chintapalli, K.N. (2008) Benign Renal Neoplasms in Adults: Cross-Sectional Imaging Findings. American Journal of Roentgenology, 190, 158-164. https://doi.org/10.2214/AJR.07.2724 https://www.ajronline.org/doi/full/10.2214/AJR.07.2724

[7] Al-Maghrabi, H.A. and Al Rashed, A.S. (2017) Challenging Pitfalls and Mimickers in Diagnosing Anastomosing Capillary Hemangioma of the Kidney: Case Report and Literature Review. American Journal of Case Reports, 18, 255-262. https://doi.org/10.12659/AJCR.902939 https://www.ncbi.nlm.nih.gov/pmc/articles/PMC5362024/

[8] Zheng, L.P., Shen, W.A., Wang, C.H., Hu, C.D., Chen, X.J., Shen, Y.Y. and Wang, J. (2020) Anastomosing Hemangioma Arising from the Left Renal Vein: A Case Report. World Journal of Clinical Cases, 8, 4986-4992.

https://doi.org/10.12998/wjcc.v8.i20.4986 https://www.wjgnet.com/2307-8960/full/v8/i20/4986.htm

[9] Zhao, M., Li, C., Zheng, J. and Sun, K. (2013) Anastomosing Hemangioma of the Kidney: A Case Report of a Rare Subtype of Hemangioma Mimicking Angiosarco$\mathrm{ma}$ and Review of the Literature. International Journal of Clinical Experimental Pathology, 6, 757-765. https://www.ncbi.nlm.nih.gov/pmc/articles/PMC3606867/

[10] Rodrigues, M.A.S., Fonseca, E.K.U.N., Yamauchi, F.I. and Baroni, R.H. (2017) Anastomosing Hemangioma Simulating Renal Cell Carcinoma. International Bra- 
zilian Journal of Urology, 43, 987.

https://doi.org/10.1590/s1677-5538.ibju.2016.0653

[11] Omiyale, A.O. (2015) Anastomosing Hemangioma of the Kidney: A Literature Review of a Rare Morphological Variant of Hemangioma. Annals of Translational Medicine, 3, 1-8. 